\title{
ENHANCING ABDUCTIVE REASONING IN DESIGN AND ENGINEERING EDUCATION VIA PROBABILISTIC KNOWLEDGE; A CASE STUDY IN AI
}

\author{
Fernando GALDON, Ashley HALL and Laura FERRARELO \\ Royal College of Art
}

\begin{abstract}
As we are moving into a knowledge-based economy, frameworks addressing the translational processes around value and impact permeate the development of educational curriculums in the design and engineering educational spectrum. In response to this approach, this paper presents an operational framework that explores how abductive reasoning, and its embodied probabilistic knowledge can bridge the gap between the challenges of accelerating technological development and current design and engineering educational practice. This is to enable students to locate, evaluate and work creatively with knowledge to generate new and improved solutions that can tackle uncertain and future real-world challenges, while delivering impact and value for society. In the process, we introduce probabilistic knowledge as the most adequate model to translate potentialities into impact and value. This repositioning enables practitioners to move beyond proving reality to a generative space aiming to transform it. In this context we present abductive reasoning as a fundamental approach to deal with directional and transformational potentialities to tackle uncertainties.
\end{abstract}

Keywords: Abductive reasoning, prospective, probabilistic knowledge, innovation research

\section{INTRODUCTION}

As we are transitioning into a knowledge-based economy, frameworks addressing the translational processes around value and impact permeate the development of educational curriculums in the design and engineering educational spectrum. In response to this approach, this paper presents an operational framework that explores how abductive reasoning, and its embodied probabilistic knowledge can bridge the gap between the challenges of accelerating technological development and current design and engineering educational practice.

In the 1970s, John Chris Jones postulated that design was different from the arts, sciences, and mathematics.

"The main point of difference is that of timing. Both artists and scientists operate on the physical world as it exists in the present (whether it is real or symbolic), while mathematicians operate on abstract relationships that are independent of historical time. Designers, on the other hand, are forever bound to treat as real that which exists only in an imagined future and have to specify ways in which the foreseen thing can be made to exist." [1]

As stated in the quote, he understood design as a future-led form of prospective thinking activity in the context of abductive reasoning in which the designer is making decisions without having all the information. Similarly, Dorst [2] and more recently Cramer-Petersen et al. [3] have concluded that design combines deductive and abductive reasoning.

This intrinsic prospective approach of design, based on abductive reasoning, planning, solution-based problem solving, problem shaping, synthesis, preparedness, readiness, and appropriateness in the built environment, determines a different model of knowing (Fig. 1). In this scenario, the designer is dealing with wicked problems by accessing areas yet-to-be or not-fully-formed [5]. Consequently, its output is based on potentialities, not certainties. We trade some degree of accuracy for access to areas that are yet-to-be or not-fully-formed. Therefore, our output is probabilistic, and research is always preliminary in its nature. Moreover, in exchange we provide guiding knowledge for prospective technological developments - as Glanville proposed, 'knowledge for' future action and possibilities rather than 'knowledge of' past actions and events [4]. Design practise is directional and transformational at its core. 
In this context, we are more concerned with how things "ought to be'"[5:111-167] instead of how things actually are. These elements position design research as crucial for addressing the impact of the accelerating technological developments, based on technology complexity, black-box technologies, and wicked problems.

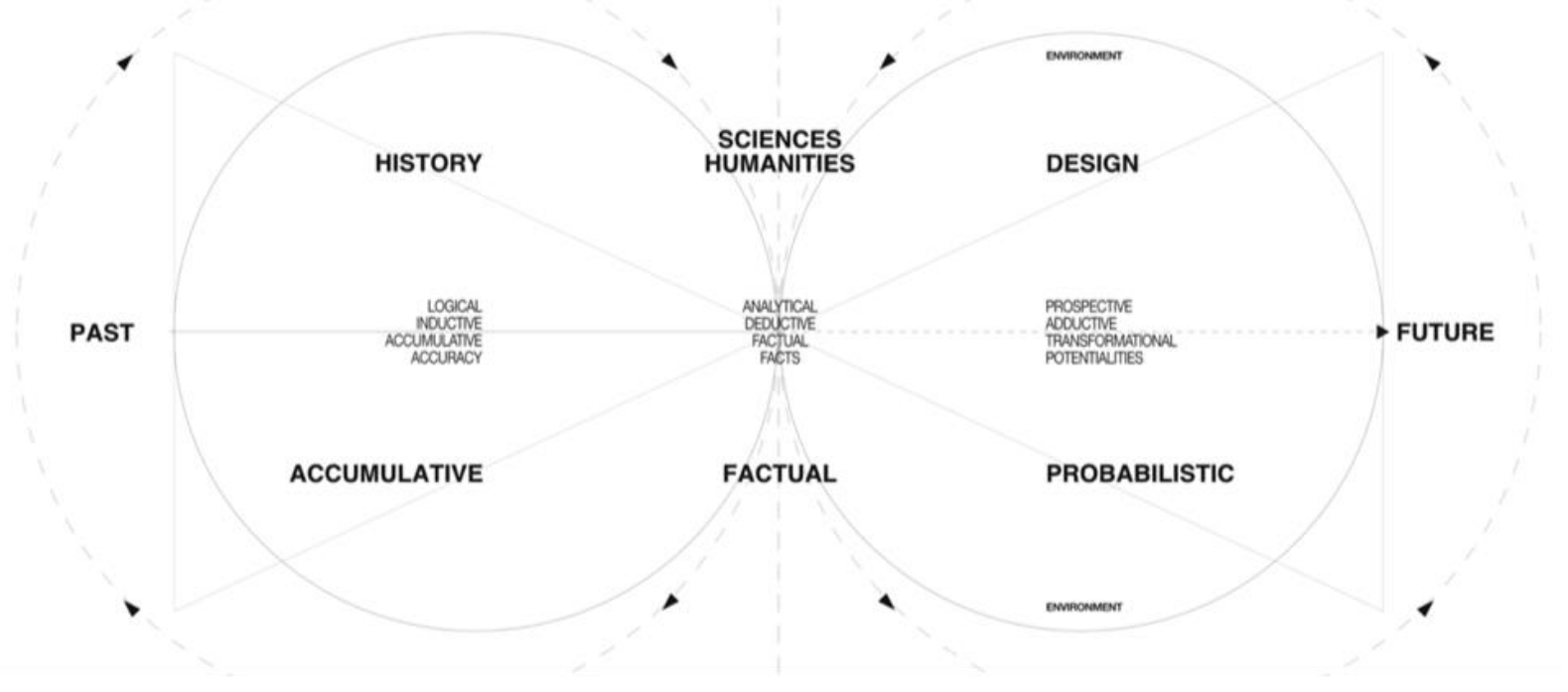

Figure 1. Prospective design research model. Fernando Galdon, 2019. [6]

\section{ABDUCTION}

An abduction process builds from an observation that aims for explaining an anomaly that is not possible to be addressed by an established theory. The abductive approach process moves, "from rule to result to case" [7:137]. It differs from deductive processes which move from rule to case to result. Or inductive logic which moves from case to result to rule. This approach is particularly helpful in the first stages of the research process, which is concerned with the formulation and selection process of hypotheses/propositions. In this context, abductive research helps to derive them in a way that can be tested in a final deductive phase of research. [7]

The fundamental focus of abductive reasoning is to search for a suitable theory to address a deviant/unexpected observation. This process is acknowledged by Dubois and Gadde [8] as "theory matching", or "systematic combining". In this process a learning loop is established by collecting simultaneously data and building the theory. It implies an interactive 'back and forth' directionality between theory and observation [7]. This process is similar to action research and can also be found in case study research. (Fig. 2)

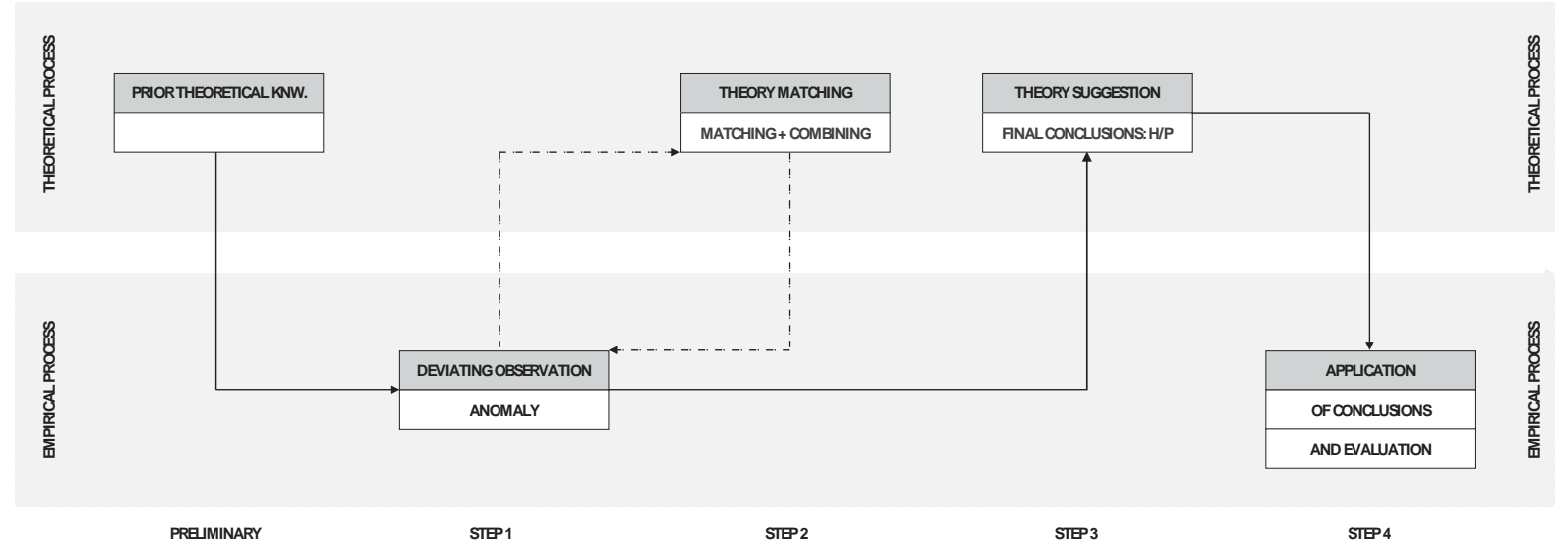

Figure 2. Abductive research framework. Galdon Building from Kovács \& Spens (2005). [7] 


\section{PROBABILISTIC KNOWLEDGE}

However, this future-led proposition presents a problem for the ontology of knowledge as currently configured. Current established practices are limited by the present, and the designer is the witness, either through measurement or observation. In this area, if we analyse what happens in economic research, we may find a suitable framework to solve this conundrum.

Economic forecasting is the process of making predictions about the economy. Economists use a statistical analysis of historical data to determine a forecast. Formal forecasts are produced yearly. However, quarterly updates or corrections are implemented to fine-tune the projection. The fundamental function of the economist is to anticipate future risks (i.e., events or conditions that can cause the result to vary from their initial estimates). These forecasts are continuously updated as the conditions of the environment evolve. These evolutions determine whether the adjustments will get tighter or looser, and how interest rates will vary, affecting a wide range of factors from loan repayments to employment levels.

This type of knowledge can be characterised as probabilistic knowledge of the future. Based on these economic forecasts, international institutions and governments infer value propositions. From this perspective, economic research enables design to access the future by legitimising probabilistic knowledge as a valid type of knowledge. This element provides a bridge to reconcile the probabilistic and abductive nature of design with established frameworks of practise that have so far been understood as factual [8]. This type of knowledge may facilitate how design and engineering students may address future challenges without fully knowing what they are.

The fundamental difference between economics and policy-making and design research is the directionality of the action. In design research we use this preliminary knowledge to co-shape the future. Design allows us to be proactive and move towards more imminent future transformations. The role of the designer is this process is to direct technological developments and reduce future risks to improve people's lives. (e.g., AI)

\section{Discussion}

We tested this process through a case study investigating the changing and impactful nature of AI from a Machine Learning (ML), Deep Learning (DL), and Reinforcement Learning (RL) developments perspective via a Quasi-Experimental Non-equivalent Control Group Post-Test-Only Design. This experiment was conducted with postgraduate students from the RCA. Their diversity in terms of background, programme of study, culture and nationality, and their diverse, critical, and enabling capabilities, plus the unifying element of being designers, provided an ideal group of participants to develop the task at hand. Then, we implemented a cross-disciplinary and progressive evaluation process via peer-review academic conferences to infer transversality and robustness.

The process of abductive reasoning started when an observation in the early stages of the process did not match existing theories [9]. In the case study we conducted, this process started when we realised that the nature of $\mathrm{AI}$ was changing due to ML/DL/RL new development and implementations, therefore we needed a new theory to design its main elements - uncertainty and trust. Then, a creative iterative process of "theory matching" or "systematic combining" started in an attempt to find a new matching framework. The aim of this process was to understand the new phenomenon to suggest a new theory in the form of new hypotheses or propositions. This process led to figuring out a range of methods to address the nature of these emerging systems. This process constructed a theory of Prospective Design (PrD), which then was applied via a case study on Virtual Assistants (VA). The development and implementation process consisted of a combination of archival research, surveys, co-design activities, and workshops. These processes inserted consequential analyses to enable ethical directionalities in its development. (See [6] for detailed results)

In order to evaluate the output, we implemented an adaptation of the Quasi-Experimental multiple group post-test-only design, also known as Non-equivalent Control Group Post-Test-Only Design. In this type of design, the control group is non-equivalent, meaning that "participants are not assigned to either the experimental or the control group in a random manner" [10]. The pre-test was unnecessary to establish equivalence between groups, as all participants were design students from the same department and institution, and the workshops were both about the future technological development of VAs. In this context, the first workshop introduced a simplified version of the methodology (fig. 3), (control group) whereas the second workshop introduced an expanded version of model (Fig. 4). 


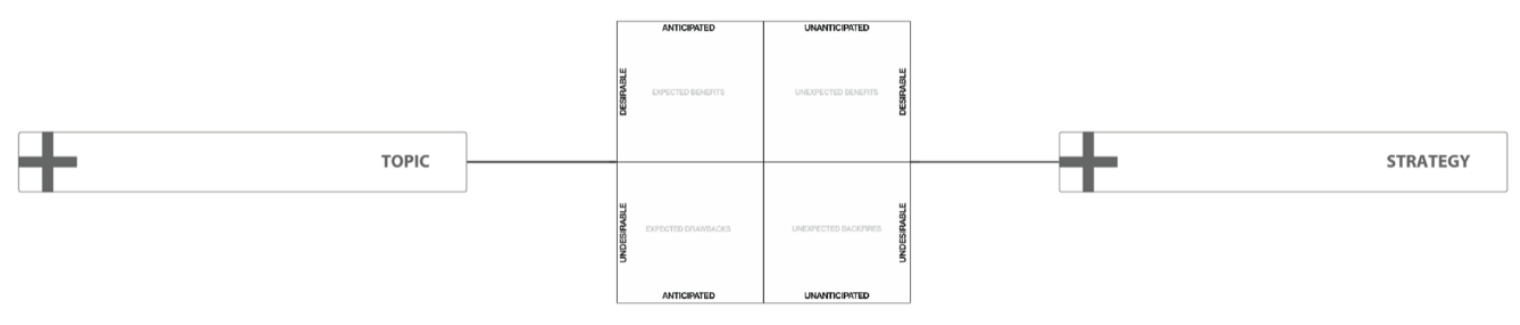

Figure 3. Consequential analysis toolkit. [6]
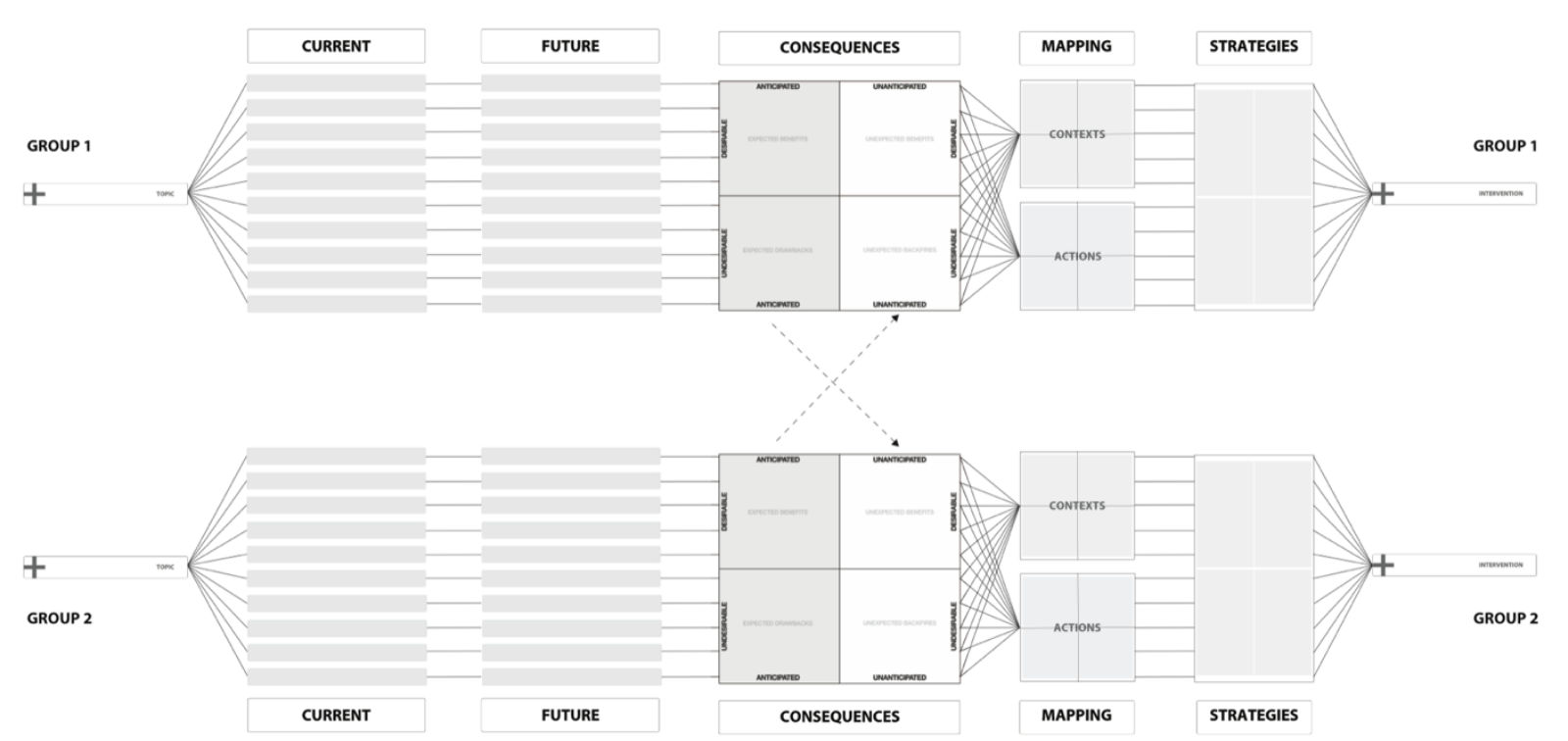

The workshops aimed to understand whether prospective insights could be transformed into applied ethical interventions and grounded in the real world by applying a systematic analysis between the insight and the design activity to design trusted systems (Fig. 4). The simplified model generated reactive responses, whereas the full model created proactive responses.

In the second workshop, participants were asked to conduct the consequences quadrant used in Workshop 1, then each group mapped the anticipated desired and undesired, and by confronting both groups, the unanticipated emerged for each group (See [6] for detailed results and qualitative analysis). This element presented participants with their own limitations and enhanced self-criticality. They then mapped the prospective outcomes in terms of the impact on contexts and the impact of actions. This analytical step allowed them to understand the impact of contexts and actions on users. Finally, participants were asked to complete a design activity consisting of developing preventive strategies for the potentially harmful and power-asymmetric interactions they had mapped. They were requested to use counter-fictional principles to transform the dystopic into real-world strategies that could be applied. The results were successful, and presented strategies aiming to ground prospective insights in potential real-case interventions that aimed to reverse asymmetries to build trust. The author(s) initial research method focussed on qualitative research [6], however this research explores additional possibilities identified through a quantitative approach in order to frame future directions.

\subsection{Future directions}

As design and engineering education is transitioning from practise (output) to research (impact) questions of evaluation in the context of abductive reasoning emerged. How could we evaluate innovation when implementing abducting reasoning in the context of impact? In this context we present a potential framework to address this emerging reality based on work conducted as part of a $\mathrm{PhD}$ implementing abductivity and probabilism. In order to address the abductive process and its emerging theory and practice, a cross-disciplinary and progressive evaluation process was implemented via peerreview academic conferences (Fig. 5). 


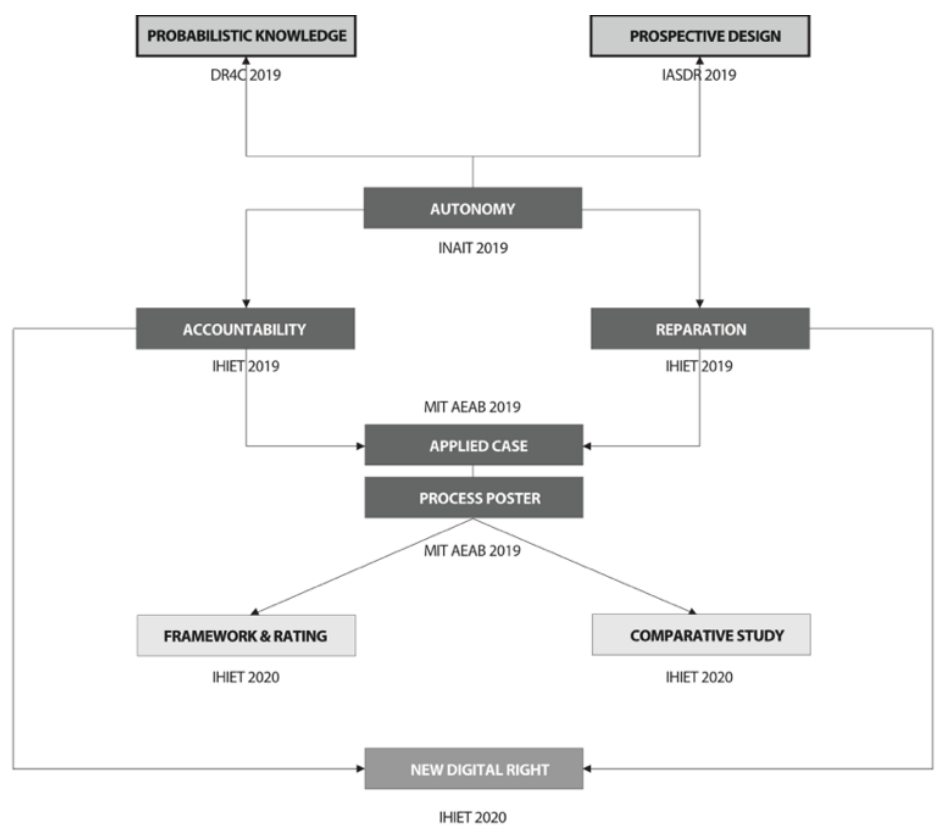

IHIET 2020

Figure 5. This table represents the progressive and cross-disciplinary publishing strategy implemented to generate robustness in the enquiry

The cross-disciplinary publishing strategy aimed to integrate diversity, transversality, impact, relevance, and responsibility in the process. As a result, we published ten papers in five different fields, ranging from Industry 4.0, human factors and design research to design futures, or applied engineering. This approach to practice aimed to support the design interventions and theoretical framework and enhance the impact of the research in terms of outputs and scrutiny by diverse audiences to maximise its transversality and therefore, its robustness. This model aims to facilitate a potential transition to impactled practise, as well as affect and inspire educational models in design and engineering in breaking its silos and inserting more transdisciplinary in the development of their processes, curriculums, cohort formation, and/or faculty.

\section{CONCLUSIONS}

In this paper we introduced abductive reasoning and probabilistic knowledge as means to address notions of prospectivity and innovation via a case study on AI.

In the insights presented, the students implementing the full methodology understood, deployed, and tested both abductive thinking and the concept of prospective design and gained a process to deal with uncertainty and the ethical impact of technological developments. Consequently, we suggest the need to include prospective ethical frameworks in design to involve students in ethical issues: to go beyond what already exists, as well as beyond the positive impact of technology and design strategies to address and/or mitigate unintended consequences, as they are fundamental for the optimum development of society; to propose that things can be otherwise. In this context, probabilistic knowledge provides a bridge to reconcile the future-led and abductive nature of design. In the process, this work challenges and develops current notions in design education based on technological progress to a model based on ethical responsibility, which places equal value on the process of design and the impact of the system on society. In this context, abductive thinking becomes the primary design mindset in driving the transition from current to potential states, leading to the mediation of anticipated and non-anticipated consequences. Finally, the implementation of a Quasi-Experimental evaluation test inserted flexibility into the process, however, it also inserted preliminary. This element was addressed with a crossdisciplinary and progressive evaluation process via peer-reviewed academic conferences.

This work has been implemented in the context of sessions. Future work will be dedicated to adapting this framework to modular structures as a testbed to articulate a full academic curriculum.

\section{REFERENCES}

[1] Jones J. C. (1992). Design methods. New York: Van Nostrand Reinhold.

[2] Dorst K. (2010). The nature of design thinking. In: DTRS8 Interpreting Design Thinking: Design Thinking Research Symposium Proceedings, 2010, pp. 131 - 139. 
[3] Cramer-Petersen C. L., Christensen B. T., and Ahmed-Kristensen S. (2019). Empirically analysing design reasoning patterns: abductive-deductive reasoning patterns dominate design idea generation. Design Studies, 60, 39-70. DOI: 10.1016/j.destud.2018.10.001.

[4] Glanville R. (2005). Design propositions. In: M. Belderbos and J. Verbeke, eds. The unthinkable doctorate: Brussels: Sint Lucas.

[5] Simon H. (1996). The sciences of the artificial. Cambridge, MA: MIT Press.

[6] Galdon F., Hall A., and Wang S. J. (2019). Prospective design: A future-led mixed-methodology to mitigate unintended consequences. In: Proceedings of the IASDR2019, The University of Manchester, UK.

[7] Kovács G. and Spens K. M. (2005), "Abductive reasoning in logistics research", International Journal of Physical Distribution \& Logistics Management, Vol. 35 No. 2, pp. 132-144.

[8] Galdon F. and Hall A. (2019). The ontological nature of design; prospecting new futures through probabilistic knowledge. Design Research for Change Symposium. Design Museum, London.

[9] Dubois A. and Gadde L.-E. (2002), "Systematic combining: an abductive approach to case research", Journal of Business Research, Vol. 55, pp. 553-60.

[10] Jackson S. L. (2009). Research methods and statistics': a critical thinking approach. Third edition. Wadsworth: Cengage Learning. 